\title{
Synthesis of silver nanoparticles by sophorolipids: Effect of temperature and sophorolipid structure on the size of particles ${ }^{\dagger}$
}

\author{
M B KASTURE, P PATEL, A A PRABHUNE, C V RAMANA, A A KULKARNI* and \\ B L V PRASAD* \\ National Chemical Laboratory, Dr. Homi Bhabha Road, Pune 411008 \\ e-mail: aa.kulkarni@ncl.res.in; pl.bhagavatula@ncl.res.in
}

\begin{abstract}
We report in situ synthesis of silver nanoparticles using biosurfactants called sophorolipids as reducing and capping agents. We further study the effect of temperature and the structure of sophorolipid on the size of silver nanoparticles obtained. The silver nanoparticles were characterized by UVvisible, transmission electron microscope (TEM) and light scattering (DLS) analysis techniques.
\end{abstract}

Keywords. Sophorolipid; nucleation and growth; dynamic light scattering; silver nanoparticles.

\section{Introduction}

Physicochemical properties of metal nanoparticles have a strong dependence on their shape ${ }^{1}$ and size, ${ }^{2}$ which make them strong candidate for various applications such as catalysis, ${ }^{3}$ biosensing, ${ }^{4,5}$ recording media ${ }^{6}$ and optics. ${ }^{7}$ Silver nanoparticles have been widely studied due to their applications of optical properties $^{8}$ and application such as antimicrobial agent, ${ }^{9,21}$ substrate for surface enhanced Raman spectroscopy (SERS) ${ }^{10}$ and biosensing. ${ }^{11}$ Their aforementioned interesting properties and enormous application potential has led to numerous methods being developed for synthesis of nanoparticles of various shapes and sizes. Typically, the methods employed for the synthesis of these nanomaterials include solution based method, ${ }^{12}$ micelle, ${ }^{13,14}$ sol gel, ${ }^{15}$ chemical precipitation, ${ }^{16}$ hydrothermal synthesis, ${ }^{17}$ pyrolysis $^{18}$ and vapour deposition. ${ }^{19}$

Nowadays, molecules, which exhibit dual nature such as capping and reducing in one go, ${ }^{20}$ are preferred since the reaction takes place in one step and thereby the need for external reducing agents is eliminated. This may enable us to exert greater control on the reaction parameters and condense the number of steps involved in synthesis so that the lab scale syntheses could be scaled up greatly enhancing their application potential. Another focused area of research is accomplishing the nanoparticle formation

${ }^{\dagger}$ Dedicated to Prof. C N R Rao on his 75th birthday

*For correspondence in the aqueous media. ${ }^{21}$ This is also an important stipulation from the environmental point of view if the large scale nanoparticle synthesis needs to be accomplished. The great hurdle to overcome in the large scale synthesis of nanomaterials, though, is the concentrations of reagents that we can employ. Since, the higher concentrations may lead to irreversible aggregations; the synthesis is always performed in $\mathrm{mM}$ concentrations. A way forward from this stumbling block is the usage of continuous flow reactions $^{22}$ where again molecules that can accomplish reduction and capping in one go are preferred. However, a thorough understanding of the various factors that lead to the formation of nanoparticles with desired parameters (size, shape, particle size distribution, etc.) is needed to convert a laboratory batch process into a continuous process.

As a first step towards the above aim, here we report the effect of different parameters that control the synthesis of the silver nanoparticles under consideration. Using sophorolipids we have recently started exploring the utility of bio-surfactant called sophorolipid as capping and reducing agent in nanoparticle synthesis. ${ }^{23}$ These have been shown to result in the reduction of $\mathrm{Ag}^{+}$ions to $\mathrm{Ag}$ nanoparticles and cap the ensuing nanoparticles. Sophorolipids are molecules with a sophorose - a dimeric glucose attached to $\omega$ or $\omega-1$ carbon of fatty acids (oleic acid, stearic acid, etc.). The final sophorolipid capped nanoparticles that we obtain fall in the category of glyconanoparticles. These glyconanoparticles are being investigated with great fervour these days for their widespread applications as cell mimicks, to 
understand protein-carbohydrate interactions and in various biomedical applications also. ${ }^{24}$ Here in the current study, we present the results on particle size variation as a function of time and temperature when two different types of sophorolipids are used for the synthesis of Ag NPs. The objective is to identify the suitable conditions that yield specific particle sizes with other reaction parameters being the same. The understanding from these results will have great bearing on further work where these reactions will be carried out in continuous flow processes. The nanoparticles are characterized by UV-visible, transmission electron microscopy and dynamic light scattering (DLS) studies.

\section{Experimental}

Silver nitrate and potassium hydroxide were purchased from SRL and Merck respectively. Oleic acid and linoleic acid were purchased form Aldrich and the chemicals used as media in synthesis of sophorolipid like malt extract, glucose, yeast extract and peptone were purchased from $\mathrm{Hi}$ Media. All the chemicals were used as received without further purification.

\subsection{Characterization techniques}

2.1a UV-visible: UV-visible measurements were carried out on Jasco V-570 UV/VIS Spectrophotometer at the resolution of $1 \mathrm{~nm}$.

2.1b Transmission electron spectroscopy: TEM measurements were performed on FEI model Technai $G^{2} F-30$ operated at an accelerating voltage of $300 \mathrm{kV}$.

2.1c Dynamic light scattering measurements: DLS measurements were carried out on Brookhaven Instrument model 90 Plus Particle Size Analyzer.

\subsection{Methodology}

The preparation of sophorolipids from oleic acid and linoleic acid was carried out following reported procedures $^{25}$ and the spectroscopic characterization concurs with the reported data. ${ }^{23}$

Synthesis of sophorolipid capped Ag NPs was carried out at different temperatures. In typical experiment when one or two drops of concentrated
$\mathrm{KOH}$ solution is added to $10 \mathrm{~mL}$ of solution containing sophorolipid $\left(1 \times 10^{-3} \mathrm{M}\right)$ and $\mathrm{AgNO}_{3}\left(1 \times 10^{-3} \mathrm{M}\right)$ colour of the solution changes to yellow indicating the formation of $\mathrm{Ag}$ nanoparticles. This experiment was repeated at five different temperatures viz. 30 (room temperature; RT), 40, 60, 80 and $90^{\circ} \mathrm{C}$ and with two different sophorolipids viz. oleic acid and linoleic acid derived sophorolipid for a period of $5 \mathrm{~min}$. The temperature was maintained constant using a thermostat (Julabo, Germany). For the characterization using light scattering, the experiments were carried out at above-mentioned different temperatures while the solution was kept at that temperature inside the light scattering experiment also using a thermostat. Control experiments carried out in exactly the same manner described above but with pure oleic acid and linoleic acid molecules did not lead to the formation of AgNPs indicating that the sophorose moiety is necessary for the reductive synthesis of AgNPs.

\section{Results and discussion}

Scheme 1 shows the structures of oleic acid (A), oleic acid sophorolipid (B), linoleic acid (C), and linoleic acid sophorolipid (D). Oleic acid (OA) has one cis double bond while the linoleic acid (LOA) has two cis double bonds.

UV-Visible spectra were recorded for Ag NPs synthesized using $\mathrm{OA}$ and LOA sophorolipid as capping/reducing agent. Figure 1A shows the UVvisible spectra for OA-sophorolipid capped/reduced Ag NPs synthesized at different temperatures while figure 1B corresponds to LOA sophorolipid reduced/ capped Ag NPs. Curves 1-5 corresponds to different temperatures employed i.e. $30^{\circ} \mathrm{C}, 40^{\circ} \mathrm{C}, 60^{\circ} \mathrm{C}, 80^{\circ} \mathrm{C}$ and $90^{\circ} \mathrm{C}$ respectively. In both the cases $\mathrm{Ag}$ NPs display a very distinct yellowish-brown colour and an absorbance in the region $400-420 \mathrm{~nm}$ accredited to the collective oscillations of conduction electrons and termed as surface plasmon resonance (SPR). ${ }^{26}$ From the UV-visible curves we observe that at room temperature no distinct surface plasmon resonance peak is observed when both OA and LOA derived sophorolipids are used as reducing and capping agents. As the temperature increases surface plasmon peak begins to evolve and at $90^{\circ} \mathrm{C}$ a well defined and sharp peak is observed. This trend is same in both OA and LOA cases; however in case of LOA derived sophorolipid no clear SPR is observed up to $80^{\circ} \mathrm{C}$ while at $90^{\circ} \mathrm{C}$ it becomes very apparent. Figure 

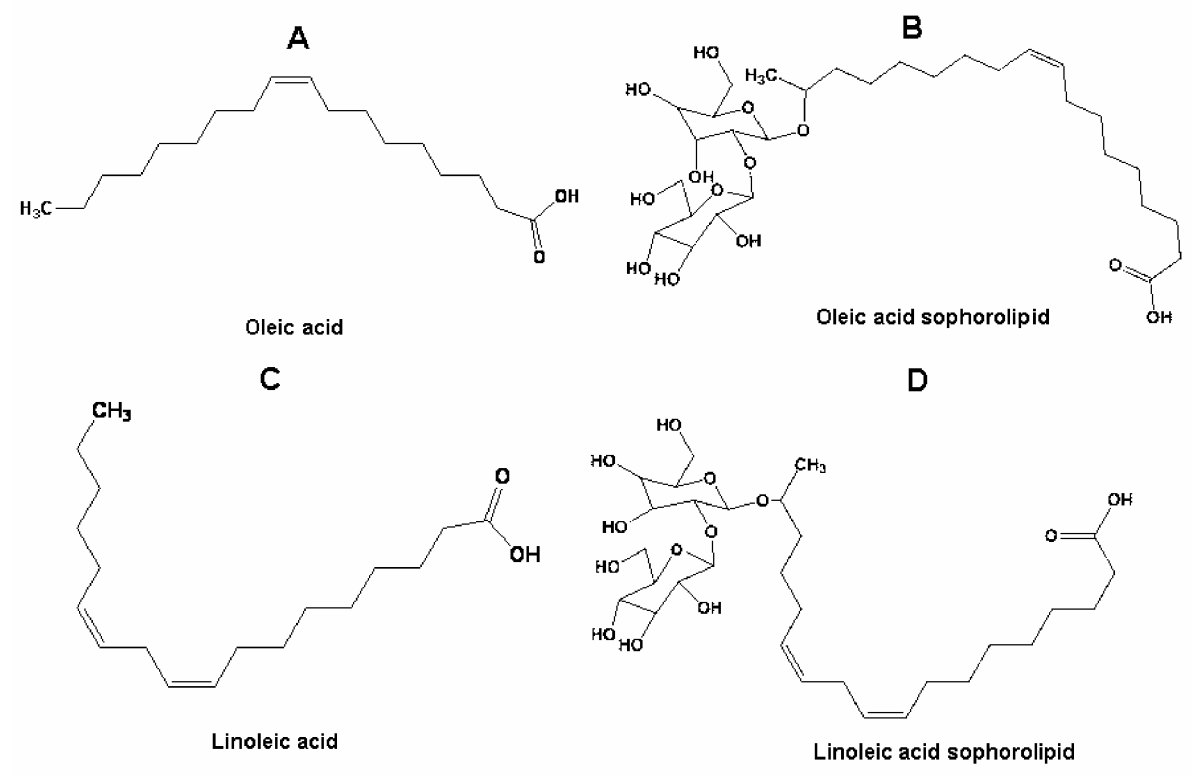

Scheme 1. Structure of oleic acid (A), oleic acid sophorolipid (B), linoleic acid (C) and linoleic acid sophorolipid (D).
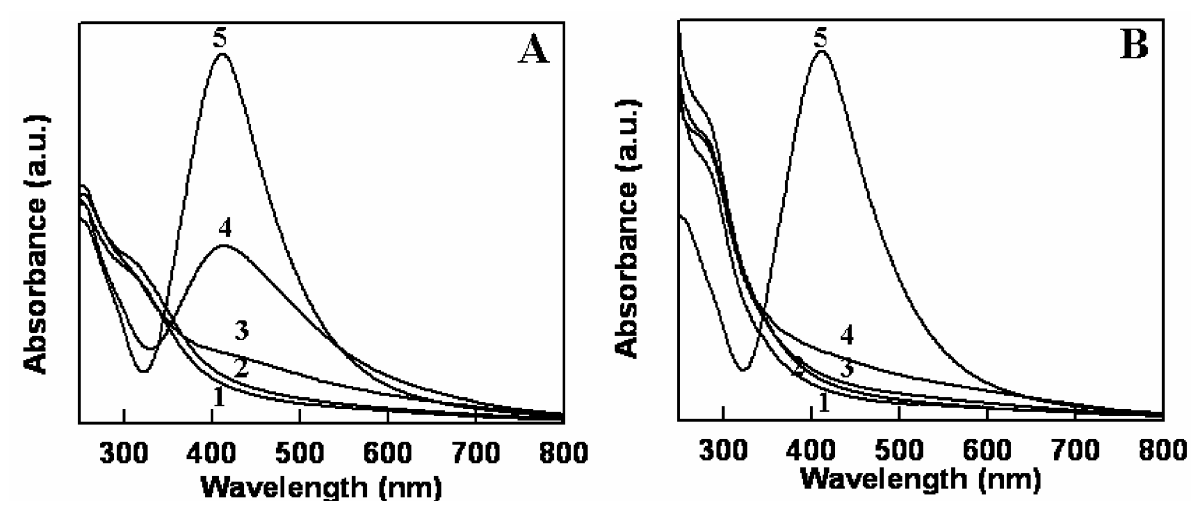

Figure 1. Shows UV-visible spectra for silver nanoparticles synthesized using OA sophorolipid (A) and LOA sophorolipid (B) as capping and reducing agent. Curves 1-5 were recorded from the samples at different temperatures at which the experiments were carried out in both cases.

2A represents the TEM image of OA sophorolipid capped/reduced $\operatorname{Ag}$ NPs synthesized at $40^{\circ} \mathrm{C}$. It is observed from the TEM images that the particles are poly-dispersed. The particle size distribution is shown in figure $2 \mathrm{~B}$ and the estimated mean particle size is close to $20 \mathrm{~nm}$. Figure 2D shows the TEM image for OA sophorolipid capped/reduced Ag NPs synthesized at $90^{\circ} \mathrm{C}$. In this case, the particles are spherical almost mono-dispersed and also smaller in size than the particles synthesized at $40^{\circ} \mathrm{C}$. The average particle size in this case is $5.5 \mathrm{~nm}$ (figure 2E). For LOA sophorolipid similar trends are observed. Figure $3 \mathrm{~A}$ shows TEM image of LOA capped/reduced Ag nano- particles synthesized at $40^{\circ} \mathrm{C}$. Particles are again polydispersed with average particle size as $22 \mathrm{~nm}$ (figure 3B). For reaction carried out at higher temperature i.e. $90^{\circ} \mathrm{C}$ particles are spherical and well defined with average particle size of $11 \mathrm{~nm}$ (figure 3E).

Particle size distribution obtained from dynamic light scattering (DLS) is shown in figure $2(\mathrm{C}, \mathrm{F})$ and in figure $3(\mathrm{C}, \mathrm{F})$. Figures $2(\mathrm{C}, \mathrm{F})$ represent particle size distribution for OA sophorolipid capped/reduced Ag nanoparticles at $40^{\circ} \mathrm{C}$ and $90^{\circ} \mathrm{C}$ respectively. The average particle size obtained from DLS data (35 $\mathrm{nm}$ and $3 \mathrm{~nm}$ for reactions done at $40^{\circ} \mathrm{C}$ and $90^{\circ} \mathrm{C}$ 
respectively) were compared with the results obtained by TEM analysis $(20 \mathrm{~nm}$ and $5.5 \mathrm{~nm}$ for reactions done at $40^{\circ} \mathrm{C}$ and $90^{\circ} \mathrm{C}$ respectively). Keeping in view that TEM and DLS analyse particle sizes differently (DLS analysis includes the ligand shell and determines the hydrodynamic size where as in TEM we can look at only metallic core) the values described above from DLS and TEM at these two temperatures can be considered to be in good agreement with each other. Similarly figures 3 (C, F) represent particles size distribution obtained from DLS for LOA sophorolipid as capping/reducing agent at $40^{\circ} \mathrm{C}$ and $90^{\circ} \mathrm{C}$. At these two temperatures $\left(40^{\circ} \mathrm{C}\right.$ and $90^{\circ} \mathrm{C}$ ) the average particle sizes obtained are $28 \mathrm{~nm}$ and $14 \mathrm{~nm}$, respectively. The values obtained in this case are also in accordance to the values obtained by TEM measurements. Figure 4 shows graph of average particle size versus the temperature. This information can be used further to carry out the reaction

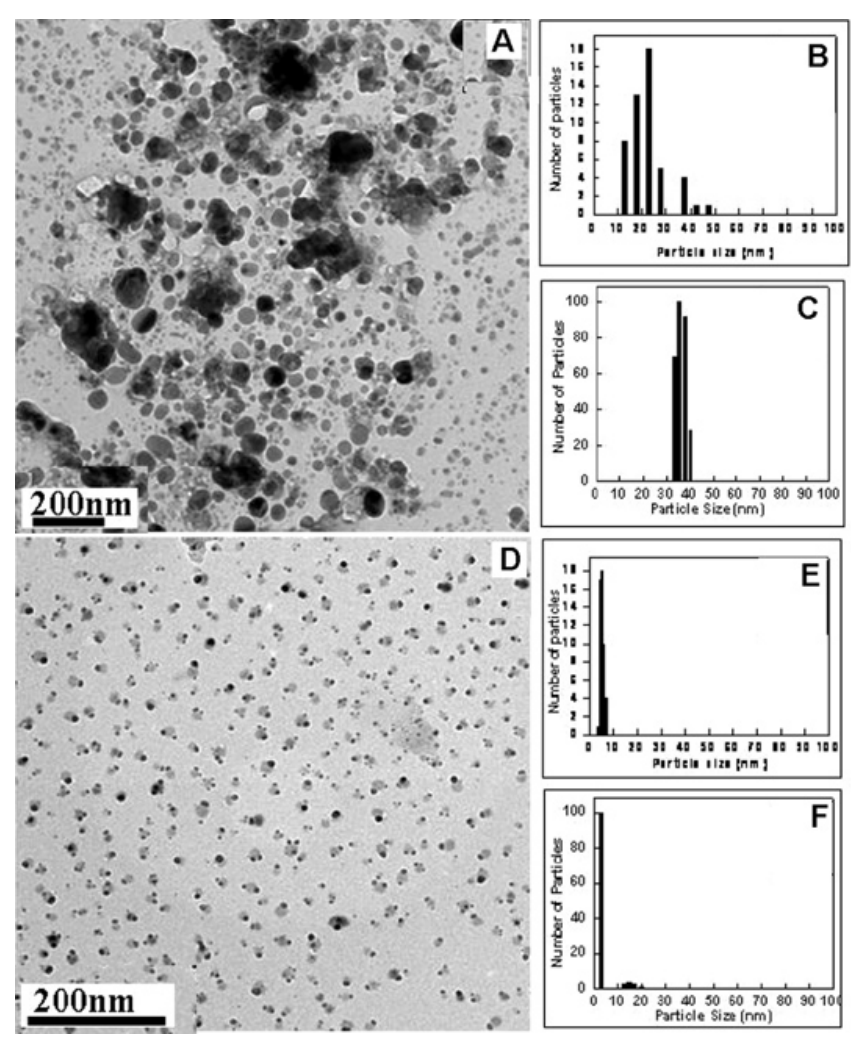

Figure 2. TEM images and particle size distribution for silver nanoparticles synthesized using OA sophorolipid as capping/reducing agent. (A) TEM image for the experiments carried out at $40^{\circ} \mathrm{C}$ and (D) TEM image for reaction carried out at $90^{\circ} \mathrm{C}$. $\mathbf{B}$ and $\mathbf{C}$ are particle size distribution form TEM and DLS respectively for reaction temperature $40^{\circ} \mathrm{C}$. $\mathbf{E}$ and $\mathbf{F}$ are particle size distribution plots obtained from TEM and DLS respectively for reaction temperature $90^{\circ} \mathrm{C}$. in continuous flow experiments. For the range of temperatures under consideration, at lower temperatures the particles are smaller and with increasing temperatures, the average particle size goes through a maximum and becomes smaller again towards higher temperature. While one would expect a continuous decrease in particle size with increase in temperature and the present result is unusual. One of the reasons for this phenomenon could be the fact that the reaction time for each case was identical. Since the reaction is relatively slow at lower temperatures, in the time for which the reaction was observed and analysed, the particles must not have formed and grown completely. While, for higher temperatures, the reaction is relatively fast and particle formation and growth is complete. In order to confirm this speculation, we carried out the synthesis at room temperature and observed the UV spectrum at every hour for almost $21 \mathrm{~h}$. It was seen that the, surface

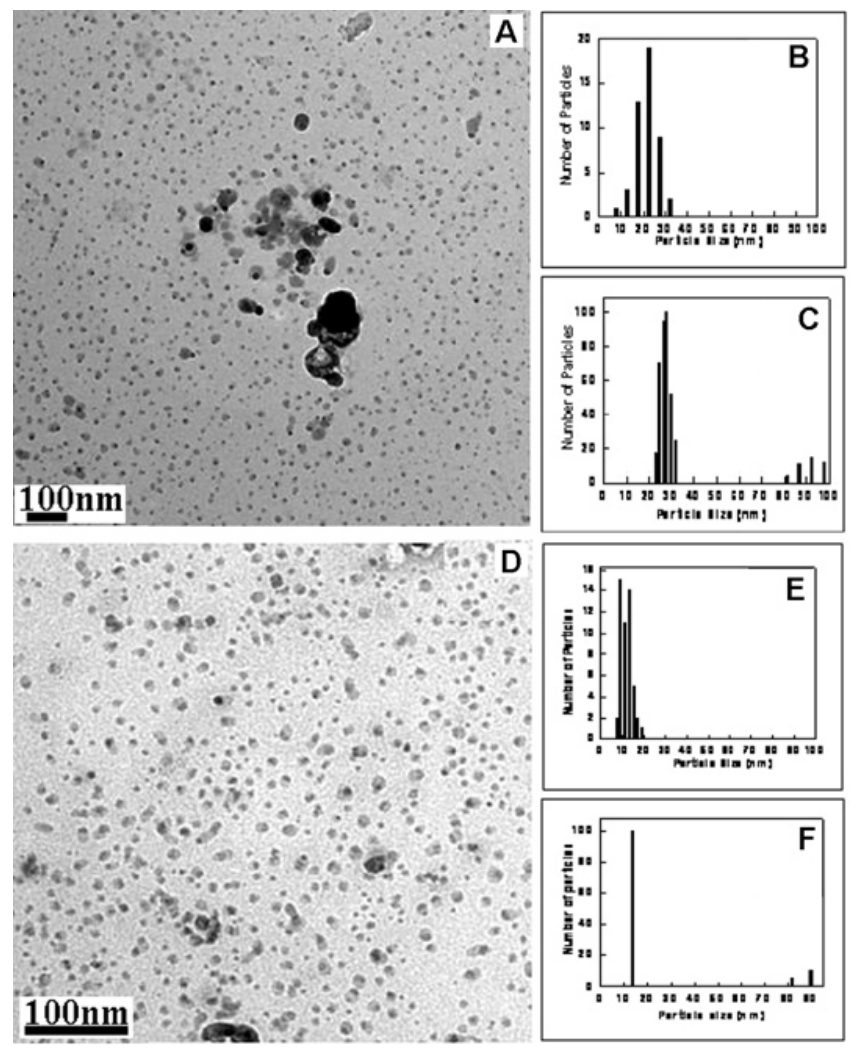

Figure 3. TEM images and particle size distribution for silver nanoparticles synthesized using LOA sophorolipid as capping/reducing agent. (A) TEM image for the experiments carried out at $40^{\circ} \mathrm{C}$ and (D) TEM image for reaction carried out at $90^{\circ} \mathrm{C}$. B and $\mathbf{C}$ are particle size distribution plots form TEM and DLS respectively for reaction temperature $40^{\circ} \mathrm{C}$. $\mathbf{E}$ and $\mathbf{F}$ are particle size distribution plots obtained from TEM and DLS respectively for reaction temperature $90^{\circ} \mathrm{C}$. 
plasmon peak was steadily increasing with time and at $21 \mathrm{~h}$, it was the highest. Thus at lower temperatures particle formation process is slow and this would certainly lead to smaller particles in the initial period of observation thereby showing a trend that passes through a peak. The observations from longer time clearly suggest that we would observe a steady decrease in the particle size with increasing temperature. This observation was evident for both the sophorolipids.

The decreased particle size and narrower size distribution with increasing temperature is a well known phenomenon. ${ }^{27}$ It is normally attributed to the increased reaction rates at higher temperatures. As the reaction rate increases the silver ions are consumed faster thus leaving less possibility for particle size growth and hence smaller particles and narrower size distributions at higher temperatures. The difference in particle sizes between OA derived sophorolipid and LOA derived sophorolipid is little intriguing. However, the roots for this apparently intriguing result may lie in the formation of olefin-silver ion complex formation. It is a well established fact that olefin silver complex depends on various factors such as chain length of olefins and on increasing number of substituents at the double bond. The stability of this complex decreases in the order $\mathrm{R}-\mathrm{CH}=\mathrm{CH}_{2}>\mathrm{R}_{2} \mathrm{C}=\mathrm{CH}_{2}>$ cis $\quad \mathrm{R} . \mathrm{CH}=\mathrm{CH} . \mathrm{R}>$ trans $\mathrm{R} . \mathrm{CH}=\mathrm{CH} . \mathrm{R}>\mathrm{R}_{2} \cdot \mathrm{C}=\mathrm{CH} . \mathrm{R}>\mathrm{R}_{2} \mathrm{C}=\mathrm{CR}_{2}{ }^{28}$ In our case we use $\mathrm{OA}$ and LOA to prepare the sophorolipid.

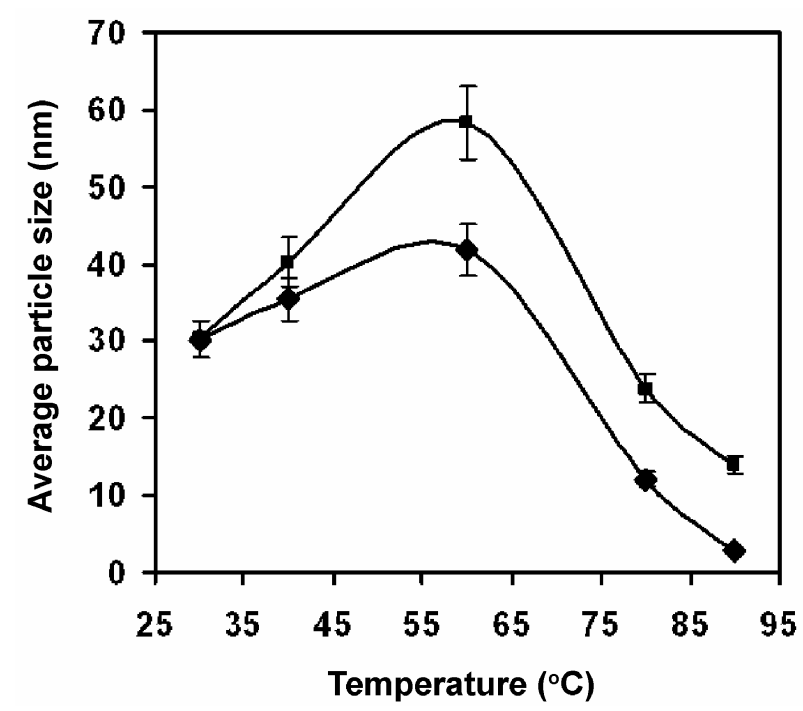

Figure 4. Average particle sizes obtained form DLS data for OA sophorolipid (1) and LOA sophorolipids reduced/capped (2) silver nanoparticles.
While OA has one cis double band and LOA has two cis double bonds (see scheme 1). So in case of LOA sophorolipid there is a probability that silver ions bind to the two double bonds more strongly or more number of $\mathrm{Ag}^{+}$ions bind to the two double bonds thus slowing down the reduction rate. Therefore, the LOA sophorolipid reduces $\mathrm{Ag}^{+}$ions to $\mathrm{Ag}$ nanoparticles more slowly. As mentioned previously, a slower reduction rate leads to bigger particles and broader particle size distributions. Further work on quantifying these aspects in terms of different relative rates is in progress.

Thus the main features of our above results can be summarized as follows. Both OA derived sophorolipid and LOA derived sophorolipids are capable of reducing silver ions to Ag NPs and the ensuing $\mathrm{Ag}$ NPs are capped by the sophorolipid or the oxidized form of the sophorolipid. The temperature dependent reduction of $\mathrm{Ag}^{+}$ions by both these sophorolipids presents us the following scenarios: (i) at low temperatures both these result in bigger particles (TEM and DLS results) and broad particle size distributions. Again in both cases as the temperature is raised the particle size becomes smaller as well as the distribution gets narrower and (ii) a more interesting observation is that when compared at each temperature condition, OA derived sophorolipid always results in smaller particle sizes as compared to LOA derived sophorolipid.

\section{Conclusions}

The formation of silver nanoparticles is reported using the OA derived sophorolipid and LOA derived sophorolipid which have the capability to act as reducing and capping agents in one go. The reactions are carried out at various temperature conditions and at lower reaction temperatures bigger particles with broad size distributions are observed. With increasing temperature, the particles were seen to become smaller with a narrow size distribution. The apparently intriguing result of LOA derived sophorolipid always leading to bigger particles in comparison to OA derived sophorolipid is explained on the basis of stronger complexation of silver ions to the two double bonds present in the former. This reduces the reaction rate and hence to slower nucleus formation and greater particle size growth. Efforts are underway to perform these reactions in continuous flow mode so that synthesis of these nanoparticles at relatively larger scale can be accomplished. 


\section{Acknowledgements}

M B K thanks Department of Science and Technology (DST) UNANST for financial support. This work was funded by DST-UNANST scheme and Department of Biotechnology (DBT) and is gratefully acknowledged. A A K thanks the Consortium on Microreaction Technology $(\mathrm{C} \mu \mathrm{R})$ www.ncl-india.org/cmr/ for financial support.

\section{References}

1. Alivisatos A P 1996 J. Phys. Chem. 10013226

2. (a) Jin R, Cao Y W, Mirkin C A, Kelly K L, Schatz G C and Zheng J G 2001 Science 294 1901; (b) Aizpurua J, Hanarp P, Sutherland D S, Käll M, Bryant G W and García de Abajo F J 2003 Phys. Rev. Lett. 90057401

3. Moreno-Manas M and Pleixats R 2003 Acc. Chem. Res. 36638

4. Mirkin C A, Letsinger R L, Mucic R C and Storhoff J J 1996 Nature 382607

5. Han M, Gao X, Su J Z and Nie S 2001 Nature Biotechnol. 19631

6. Sun S, Murray C B, Weller D, Folks L and Moser A 2000 Science 2871989

7. Kamat P V 2002 J. Phys. Chem. B106 7729

8. (a) Jin R C, Cao Y W, Mirkin C A, Kelly K L, Schatz G C and Zheng J G 2001 Science 294 1901; (b) Silva T J, Schultz S and Weller D 1994 Appl. Phys. Lett. 65 658; (c) Schultz S, Smith D R, Mock J J and Schultz D A 2000 Proc. Natl. Acad. Sci. USA 97996

9. (a) Sanpui P, Murugadoss A, Durga Prasad P V, Ghosh S S and Chattopadhyay A 2008 Int. J. Food Microbiol. 124 142; (b) Ruparelia J P, Chatterjee A K, Duttagupta S P and Mukherji S 2008 Acta Biomaterialia 43 707; (c) Kumar A, Vemula P K, Ajayan P M and John G 2008 Natl. Mater. 3236

10. (a) Nie S and Emory S R 1997 Science 275 1102; (b) Dick L A, McFarland A D, Haynes C L and P van Duyne R 2002 J. Phys. Chem. B106 853

11. (a) Link S and El-Sayed M A 1999 J. Phys. Chem. B103 8410; (b) Kottmann J P, Martin O J F, Smith D $\mathrm{R}$ and Schultz S 2001 Chem. Phys. Lett. 3411

12. (a) Qu L, Peng Z A and Peng X 2001 Nano Lett. 1 333; (b) Peng Z A and Peng X $2002 \mathrm{~J}$. Am. Chem. Soc. 1243343
13. (a) Lisiecki I, Billoudet F and Pileni M P $1996 \mathrm{~J}$. Phys. Chem. 100 4160; (b) Hao E, Bailey R C, Schatz G C, Hupp J T and Li S 2004 Nano Lett. 4327

14. (a) Turkevich J, Stevenson P C and Hillier J 1951 Discuss. Faraday Soc. 11 55; (b) Taleb A, Petit C and Pileni M P 1997 Chem. Mater. 9 950; (c) Barnickel P and Wokaun A 1990 Mol. Phys. 69 1; (d) Chen Z J, Qu X M, Fang F Q and Jiang L 1996 Colloids Surf. B7 173

15. Mondelaers D, Vanhoyland G, Van den Rul H, D'Haen J, Van Bael M K, Mullens J and Van Poucke L C 2002 Mater. Res. Bull. 37901

16. Spanhel L, Haase M, Weller H and Henglein A 1987 J. Am. Chem. Soc. 1095649

17. Sun Y-P, Guduru R, Lin F and Whiteside T Indian Eng. Chem. Res. 2000394663

18. (a) Cai W and Zhang L 1997 J. Phys. Condensed Matter 9 7257; (b) Maya L, Paranthaman M, Thundat T and Bauer M L 1996 J. Vac. Sci. Technol. B14 15

19. Liu Y, Zhu W, Tse M S and Shen Y 1995 J. Mater. Sci. Lett. 141185

20. (a) Si S and Mandal T K 2007 Chem. Eur. J. 13 3160; (b) Si S, Bhattacharjee R R, Banerjee A and Mandal T K 2006 Chem. Eur. J. 12 1256; (c) Bhattacharjee R R, Das A K, Haldar D, Si S, Banerjee A and Mandal T K 2005 J. Nanosci. Nanotechnol. 51141

21. Dahl J A, Maddux B L S and Hutchison J E 2007 Chem. Rev. 1072228

22. (a) Jahn A, Reiner J E, Vreeland W N, DeVoe D L, Locascio L E and Gaitan M 2008 J. Nanopart. Res. 10 925; (b) Song Y, Hormes J and Kumar C S S R 2008 Small 4698

23. (a) Kasture M, Singh S, Patel P, Joy P A, Prabhune A A, Ramana C V and Prasad B L V 2007 Langmuir 23 1409; (b) Singh S, Jaisawal S, Patel P, Prabhune A A, Ramana C V and Prasad B L V New J. Chem. 2008 (accepted)

24. de la Fuente J M and Penadés S 2006 Biochim. Biophys. Acta 1760636

25. (a) Inge $N A$, Bogaert V, Saerens K, De Muynck $C$, Develter D, Soetaert W and Vandamme E J 2007 Appl. Microbiol. Biotechnol. 76 23; (b) Shah S and Prabhune A A, 2007 Biotechnol. Lett. 29267

26. (a) Henglein A 1993 J. Phys. Chem. 97 547; (b) Mulvaney P 11996 Langmuir 12788

27. Park J, Joo J, Kwon G S, Jang Y and Hyeon T 2007 Angew. Chem. Int. Ed. 464630

28. de Ligny, C L 1976 in Advances in chromatography (eds) J C Giddings, E Grushka, J Cazes and P R Brown (New York: Marcel Dekker) vol. 14, pp 265-304 